\title{
Estimation of spark protection device's reliability influence on the fire safety of apartment's network with harmonic currents and voltage up to $1 \mathrm{KV}$
}

\author{
Pavel Kuznetsov ${ }^{1, *}$, Sergej Solyonyj ${ }^{2}$, Yuriy Sychev $^{1}$, Vladislav Shishlakov ${ }^{2}$ and Oksana Solenaya ${ }^{2}$ \\ ${ }^{1}$ Saint-Petersburg Mining University, Department of Power Industry and Electromechanics, 199106, Saint Petersburg, Russia \\ ${ }^{2}$ Saint-Petersburg State University of Aerospace Instrumentation, Department of Electromechanics and robotics, 190000 Saint \\ Petersburg, Russia
}

\begin{abstract}
This article analyses the statistical level of fire danger in living premises. Its influence on the reliability of apartments' electrical system is estimated and role of spark protection devices in this process is designated. In addition, a role of harmonic currents in the process of dangerous contact joints detection shown and described. In the conclusion, main requirements for diagnostic of apartments' low-voltage electrical networks are formulated.
\end{abstract}

\section{Introduction}

Based on the data received from [1-3] it was estimated that during the last decade there were 523833 fires. However, $43.7 \%$ of them were from exogenous sources. In other words, 228915 fires happened because of problems with electricity and $90 \%$ of this amount happened in living premises because of hot, sparkling disassembling power contacts' appearance. This number equals to 206023 fires during the past 10 years. This is why development of protective and preventive methods, organisation of technical inspections from hot, sparkling disassembling contacts is an actual problem nowadays.

\section{Condition of the problem}

Nowadays Automatic Circuit Breakers (ACB) and residual cut-off device (ISS) exist and are widely applied in the apartments' power networks. These gadgets have some weak sides. For example, they do not react for appearance of weakened sparkling power contact joints that leads to the burnout of the conductor's isolation and this, in turn, leads to the fires in living premises. Researches [4-6] propose technical solution with the help of which it is possible to disable weakened sparkling power contact joints. However, real prototype's tests have revealed the following important disadvantages:

- spectrum converter of high-frequency electromagnetic oscillations that is applied in the prototype was manufactured as a current transformer with the air gap in magnetic core. This core was produced from ferrite magnets (very hard, but fragile material very similar to ceramics in its mechanical properties). Due to this fact core's manufacturing becomes a hard-to-perform task that leads to prime cost increase and rise in price of the protective device;

- transformer's secondary winding is connected to the current relay directly (executive part). Thus, the entire device's sensitivity depends on the relay's operating current and all high-frequency current's oscillations that are induced by the sparkling weakened power joints or by the electric arc through the transient resistance and that will not reach relay's operating current value will not be detected by the device;

- installation of device in living premises may be performed simultaneously with the voltage regulators that are widely used for lightning intensity control or power systems' productivity control. Operating schemes of voltage regulators are based on the use of thyristors or semistors. These semiconductor devices distort voltage and current sin waves' shapes during their operation cycles and generate high-frequency distortions in the power network. Protective devices interpret them as sparkling of the weakened contact joints and false alarm happens;

- during power switches of the loads a transient mode can occur with the help of circuit breakers and automatic circuit breakers. These transient modes are often accompanied by the high frequency oscillations in the power network that also lead to the false alarm.

The above described disadvantages were removed in the device shown on Figure 1 described and proposed in [3]. This unit solves the problem of high-frequency oscillations determination. It divides the oscillations from dangerous sparkling weakened contact joints and all other safe semiconductor equipment based on the time of oscillations' existence in the power network. This guarantees modernized unit's reliable operation.

This modernization is achieved due to successful implementation of high-frequency signals auto selection

Corresponding author: kuznetsovpavel@inbox.ru 
unit between logical element OR and selective detector unit. Network parameters are measured via current transformers that are connected separately to each phase. The unit also contains a high-frequency filter. This simple scheme allows to separate dangerous oscillations that can cause fire from the simple ones and send an emergency signal to the fire or local department.

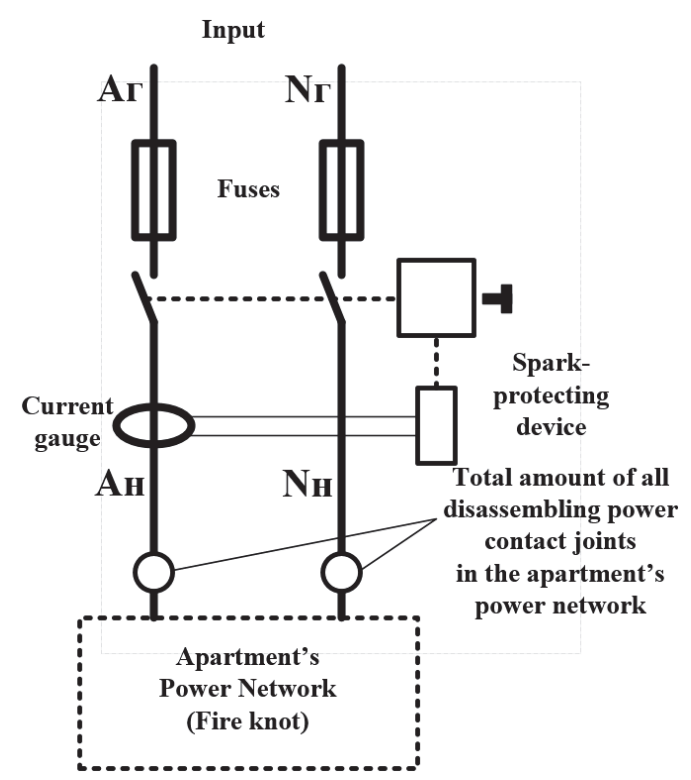

Fig. 1. Apartments' spark-protecting device.

\section{Research results}

The main aim of this article is to assess the fire safety level in living premises' power networks. It is also important to reveal influence of the sparkling protection system's reliability and its maintenance terms on the loads fire danger.

There was made an assumption that under the "fire node" definition we understand total sum of all contact joints in the apartment's power network.

In general, 19183000 danger fire nodes (apartments) are being controlled in [1-3] yearly.

The probability of yearly fire occurrence that depends on fire node must not exceed the value $Q(t)=1 \cdot 10^{-6}$, where ${ }^{t=1}$ year [4].

It is known that time intervals between fires in living premises do not conflict with exponential probability distribution function [7-9]. At that time, the year's fire rate in the premises because of weakened sparkling disassembling contact joints can be determined with the following formula:

$$
\lambda_{1}=\frac{n}{N \cdot t}
$$

where $n=50578$ is the mean fires value per year in premises because of sparkling weakened disassembling power joints; $N=19183000$ is the mean value of dangerous fire nodes in living premises (number of flats); $t=1$ year.

Probability of fire occurrence per year:

$$
Q^{*}(1)=1-e^{-\lambda_{1} \cdot t}
$$

i.e. $Q^{*}(1)=7.12 \cdot 10^{-4}$ that is 712 times higher than standardized value $Q(t)=1 \cdot 10^{-6}$.

Suppose that isolation combustion happens each time when the following accident events will coincide in time and space: appearance of weakened sparkling power joint in the apartment's electrical network with voltage up to $1 \mathrm{KV}$ and the sparkling protective device has failed to react [10].

Change of state during time in the contact joint can be presented as a homogenous random Markov process $\xi(t)$ [11-15] with two states: 0 - there are no weakened dangerously sparkling disassembling contact joints in the protected network; 1 - a weakened dangerously sparkling disassembling contact joint has appeared.

Values $\lambda_{1}$ and $\mu_{1}$ are the parameters of the process $\xi(t)$.

Sparkling protection's status change in time can be described using the same homogenous Markov process $\eta(t)$ [16-18]: 0 - sparkling protection operates normally; 1 - sparkling protection has failed. Process $\eta(t)$ has the following parameters: $\lambda_{2}$ and $\mu_{2}$.

Totality of the processes $\xi(t)$ and $\eta(t)$ can be observed as a homogenous Markov process with 4 discreet states and continuous time.

As it follows, the "contact joint - sparkling protection" system can be in one state of finite set of states:

$e_{1}(0,0)$ there are no weakened dangerously sparkling disassembling contact joints in the protected network, sparkling protection device operates normally;

$e_{2}(1,0)$ a weakened dangerously sparkling disassembling contact joint has appeared in the protecting network, sparkling protection device operates normally;

$e_{3}(0,1)$ there are no weakened dangerously sparkling disassembling contact joints in the protected network, sparkling protection device has failed;

$e_{4}(1,1) \quad$ a weakened dangerously sparkling disassembling contact joint has appeared in the protecting network, sparkling protection device has failed.

When system occasionally comes to the state $e_{4}(1,1)$ apartment's conductors isolation combustion happens in the electrical network that leads to fire in most cases.

It is possible to calculate conductors isolation's combustion probability in time:

$$
Q(t)=1-\left[P_{1}(t)+P_{2}(t)+P_{3}(t)\right]
$$

where 


$$
\left\{\begin{array}{l}
\dot{P}_{1}(t)=-\left(\lambda_{1}+\lambda_{2}\right) \cdot P_{1}(t)+\mu_{1} \cdot P_{2}(t)+\mu_{2} \cdot P_{2}(t) \\
\dot{P}_{2}(t)=\lambda_{1} \cdot P_{1}(t)-\left(\mu_{1}+\lambda_{2}\right) \cdot P_{2}(t)+\lambda_{1} \cdot P_{1}(t) \\
\dot{P}_{3}(t)=\lambda_{2} \cdot P_{1}(t)-\left(\lambda_{1}+\mu_{2}\right) \cdot P_{3}(t)
\end{array} .\right.
$$

Equation system (4) can be solved with the following initial conditions:

$$
P_{1}(0)=1, P_{2}(0)=P_{3}(0)=0 ，
$$

where $\lambda_{1}=\frac{1}{\bar{g}_{1}}, \mu_{1}=\frac{1}{g_{1}}, \lambda_{2}=\frac{1}{\bar{g}_{2}}, \mu_{2}=\frac{1}{g_{2}}$,

where

$\bar{g}_{1}$ is an average time interval between dangerous sparkling disassembling contact joint occurrence;

$g_{1}$ is an average duration of contact joint being in dangerous condition (arcing between contact joints);

$\bar{g}_{2}$ is an average time interval between failures of the spark protection system

$g_{2}$ is an average duration of the spark protection system being in an undetected failed condition.

From the equation system (4) with the help of known methods [19] we can find $P_{1}(t), P_{2}(t), P_{3}(t)$ and after their substitution in (3) we can receive combustion possibility of conductor in the apartment's power network:

$$
Q(t)=1-\frac{G\left(S_{1}\right)}{Z^{\prime}\left(S_{1}\right)} \cdot e^{S_{1} \cdot t}+\frac{G\left(S_{2}\right)}{Z^{\prime}\left(S_{2}\right)} \cdot e^{S_{2} \cdot t}+\frac{G\left(S_{3}\right)}{Z^{\prime}\left(S_{3}\right)} \cdot e^{S_{3} \cdot t}
$$

where

$$
\begin{gathered}
G(S)=S^{2}+a S+b_{1}=0, \\
Z(S)=S^{3}+a S^{2}+b S+C=0 .
\end{gathered}
$$

Roots $S_{1}, S_{2}$, and $S_{3}$ can be found from the solving of cubic equation (8) with the known methods.

$$
S^{3}+a S^{2}+b S+C=0
$$

where

$$
\begin{gathered}
a=2 \lambda_{1}+2 \lambda_{2}+\mu_{1}+\mu_{2} ; \\
b=\lambda_{1} \lambda_{2}+\left(\lambda_{1}+\lambda_{2}+\mu_{2}\right)\left(\lambda_{1}+\lambda_{2}+\mu_{1}\right) ; \\
c=\lambda_{1} \lambda_{2}\left(\lambda_{1}+\lambda_{2}+\mu_{1}+\mu_{2}\right) \\
b_{1}=2 \lambda_{1} \lambda_{2}+b .
\end{gathered}
$$

After substitution of the found roots from equation (8) we can obtain:

$$
\begin{aligned}
& Z^{\prime}\left(S_{1}\right)=3 S_{1}^{2}+2 a S_{1}+b ; \\
& Z^{\prime}\left(S_{2}\right)=3 S_{2}^{2}+2 a S_{2}+b ; \\
& Z^{\prime}\left(S_{3}\right)=3 S_{3}^{2}+2 a S_{3}+b .
\end{aligned}
$$

After substitution of $Z^{\prime}\left(S_{1}\right), Z^{\prime}\left(S_{2}\right), Z^{\prime}\left(S_{3}\right)$ and $G\left(S_{1}\right), G\left(S_{2}\right), G\left(S_{3}\right)$ values into equation (6) we obtain combustion possibility of the conductor's isolation in time $t$.

If time interval between diagnostics of spark protection system $\Theta_{2}$ is set, it is possible to find $\mu_{2}$ using equation from [20]:

$$
\mu_{2}=\frac{1}{\Theta_{2}-\frac{1}{\lambda_{2}}\left[1-e^{-\lambda_{2} \Theta_{2}}\right]} .
$$

In case if $\lambda_{2} \Theta_{2}<0,1$ :

$$
\mu_{2}=\frac{2}{\lambda_{2} \Theta_{2}^{2}}
$$

Average time before first combustion of conductor's isolation can be defined from the system of linear algebraic equations written in matrix form. This is valid only under condition that in the initial time moment system was in one of the following conditions: $e_{1}(0,0) ; e_{2}(1,0)$ or $e_{3}(0,1)$ :

$$
\begin{aligned}
& {\left[\begin{array}{l}
\tau_{1} \\
\tau_{2} \\
\tau_{3}
\end{array}\right]=\frac{1}{\lambda_{1} \lambda_{2}\left(\lambda_{1}+\lambda_{2}+\mu_{1}+\mu_{2}\right)} \times} \\
& \times\left[\begin{array}{ccc}
\left(\mu_{1}+\lambda_{2}\right)\left(\lambda_{1}+\mu_{2}\right) & \lambda_{1}\left(\lambda_{1}+\mu_{2}\right) & \lambda_{2}\left(\mu_{1}+\lambda_{2}\right) \\
\mu_{1}\left(\lambda_{1}+\mu_{2}\right) & \lambda_{1}\left(\lambda_{1}+\mu_{2}+\lambda_{2}\right) & \lambda_{2} \mu_{1} \\
\lambda_{2}\left(\mu_{1}+\lambda_{2}\right) & \lambda_{2} \mu_{2} & \lambda_{2}\left(\lambda_{1}+\mu_{1}+\lambda_{2}\right)
\end{array}\right]\left[\begin{array}{l}
1 \\
1 \\
1
\end{array}\right]
\end{aligned}
$$

From the system (11) it can be obtained:

$$
\begin{gathered}
\tau_{1}=\frac{\left(\mu_{1}+\lambda_{2}\right)\left(\lambda_{1}+\mu_{2}\right)+\lambda_{1}\left(\lambda_{1}+\mu_{2}\right)+\lambda_{1}\left(\mu_{1}+\lambda_{2}\right)}{\lambda_{1} \lambda_{2}\left(\lambda_{1}+\lambda_{2}+\mu_{2}+\mu_{1}\right)}, \\
\tau_{2}=\frac{\mu_{1}\left(\lambda_{1}+\mu_{2}\right)+\lambda_{1}\left(\lambda_{1}+\mu_{2}+\lambda_{2}\right)+\lambda_{2} \mu_{1}}{\lambda_{1} \lambda_{2}\left(\lambda_{1}+\lambda_{2}+\mu_{2}+\mu_{1}\right)}, \\
\tau_{3}=\frac{\lambda_{2}\left(\mu_{1}+\lambda_{2}\right)+\lambda_{2} \mu_{2}+\lambda_{2}\left(\lambda_{1}+\mu_{1}+\lambda_{2}\right)}{\lambda_{1} \lambda_{2}\left(\lambda_{1}+\lambda_{2}+\mu_{1}+\mu_{2}\right)},
\end{gathered}
$$

where

$\tau_{1}$ is an average time before first combustion of the conductor's isolation, if the system in the initial time moment was in the $e_{1}(0,0)$ condition;

$\tau_{2}$ is an average time before first combustion of the conductor's isolation, if the system in the initial time moment was in the $e_{2}(1,0)$ condition;

$\tau_{3}$ is an average time before first combustion of the conductor's isolation, if the system in the initial time moment was in the $e_{3}(0,1)$ condition.

Time dispersion before first combustion of conductor's isolation if the system was in one of the 
following conditions $e_{1}(0,0), e_{2}(1,0)$ or $e_{3}(0,1)$ can be found from the solution of the following algebraic equations' system written in the mathematical form:

$$
\begin{aligned}
& {\left[\begin{array}{l}
\sigma_{1}^{2} \\
\sigma_{2}^{2} \\
\sigma_{3}^{2}
\end{array}\right]=\left[\left(\begin{array}{lll}
2 & 0 & 0 \\
0 & 2 & 0 \\
0 & 0 & 2
\end{array}\right) \times\left[\begin{array}{lll}
a_{11} & a_{12} & a_{13} \\
a_{21} & a_{22} & a_{23} \\
a_{31} & a_{32} & a_{33}
\end{array}\right]-\left(\begin{array}{lll}
1 & 0 & 0 \\
0 & 1 & 0 \\
0 & 0 & 1
\end{array}\right)\right] \times} \\
& \times\left[\begin{array}{l}
\tau_{1} \\
\tau_{2} \\
\tau_{3}
\end{array}\right]-\left[\begin{array}{l}
\tau_{1}^{2} \\
\tau_{2}^{2} \\
\tau_{3}^{2}
\end{array}\right]
\end{aligned}
$$

where

$$
\begin{aligned}
& a_{11}=\frac{\left(\mu_{1}+\mu_{2}\right)\left(\lambda_{1}+\mu_{2}\right)}{\lambda_{1} \lambda_{2}\left(\lambda_{1}+\lambda_{2}+\mu_{1}+\mu_{2}\right)} ; \\
& a_{12}=\frac{\lambda_{1}\left(\lambda_{1}+\mu_{2}\right)}{\lambda_{1} \lambda_{2}\left(\lambda_{1}+\lambda_{2}+\mu_{1}+\mu_{2}\right)} ; \\
& a_{13}=\frac{\lambda_{2}\left(\mu_{1}+\lambda_{2}\right)}{\lambda_{1} \lambda_{2}\left(\lambda_{1}+\lambda_{2}+\mu_{1}+\mu_{2}\right)} ; \\
& a_{21}=\frac{\mu_{1}\left(\lambda_{1}+\mu_{2}\right)}{\lambda_{1} \lambda_{2}\left(\lambda_{1}+\lambda_{2}+\mu_{1}+\mu_{2}\right)} ; \\
& a_{22}=\frac{\lambda_{1}\left(\lambda_{1}+\mu_{2}+\lambda_{2}\right)}{\lambda_{1} \lambda_{2}\left(\lambda_{1}+\lambda_{2}+\mu_{1}+\mu_{2}\right)} ; \\
& a_{23}=\frac{\lambda_{2} \mu_{1}}{\lambda_{1} \lambda_{2}\left(\lambda_{1}+\lambda_{2}+\mu_{1}+\mu_{2}\right)} ; \\
& a_{32}=\frac{\lambda_{2}\left(\lambda_{1}+\mu_{2}+\lambda_{2}\right)}{\lambda_{1} \lambda_{2}\left(\lambda_{1}+\lambda_{2}+\mu_{1}+\mu_{2}\right)} \\
& a_{31}=\frac{\lambda_{2}\left(\mu_{1}+\lambda_{2}\right)}{\lambda_{1} \lambda_{2}\left(\lambda_{1}+\lambda_{2}+\mu_{1}+\mu_{2}\right)} ;
\end{aligned}
$$

From the equation system (15) it can be obtained:

$$
\begin{gathered}
\sigma_{1}^{2}=\left(2 a_{11}-1\right) \tau_{1}+2 a_{12} \tau_{2}+2 a_{13} \tau_{3}-\tau_{1}^{2} ; \\
\sigma_{2}^{2}=2 a_{21} \tau_{1}+\left(2 a_{22}-1\right) \tau_{2}+2 a_{23} \tau_{3}-\tau_{2}^{2} \\
\sigma_{3}^{2}=2 a_{31} \tau_{2}+2 a_{32} \tau_{2}+\left(2 a_{33}-1\right) \tau_{3}-\tau_{3}^{2},
\end{gathered}
$$

where

$\sigma_{1}^{2}$ is time dispersion before the first combustion of conductor's isolation, if the system was in $e_{1}(0,0)$ condition;

$\sigma_{2}^{2}$ is time dispersion before the first combustion of conductor's isolation, if the system was in $e_{2}(1,0)$ condition; $\sigma_{3}^{2}$ is time dispersion before the first combustion of conductor's isolation, if the system was in $e_{3}(0,1)$ condition.

Formulas $(6,12-14,17-19)$ fully characterize the fire node (apartment's network). In case, if during calculation it can be obtained that $\tau_{1} \approx \sigma_{1}, \tau_{2} \approx \sigma_{2}$, $\tau_{3} \approx \sigma_{3}$, then probability of conductor's isolation combustion can be determined with the following formula:

$$
Q_{i}(t)=1-e^{-\frac{t}{\tau_{i}}}
$$

where $i=1,2,3$.

\section{Calculation example}

\subsection{Input data}

The calculations were performed for the following initial data: $\lambda_{1}=7.12 \cdot 10^{-4} \quad 1 /$ year, $\lambda_{2}=0.2 \quad 1 /$ year, $\mu_{1}=1.576 \cdot 10^{8} 1 /$ year, $\mu_{2}=1.425 \cdot 10^{3} 1 /$ year. It is necessary to determine possibility of conductor's isolation combustion because of weakened, sparkling disassembling power contact joint appearance in the apartment's power network during the year $Q(1)$, average time before the first conductor's isolation combustion $\tau_{1}$ and dispersion $\sigma_{1}^{2}$ using formula (17), on condition that system was in condition $e_{1}(0,0)$ in the initial time moment.

\subsection{Solution}

Average time $\tau_{1}$ before the first conductor's isolation combustion can be calculated with the help of formula (12), dispersion $\sigma_{1}^{2}$ can be found using formula (17), in other words $\tau_{1}=1.0008215 \cdot 10^{7}$ years and $\sigma_{1}=1.00083 \cdot 10^{7}$ years. Because our result corresponds to the condition where $\tau_{1} \approx \sigma_{1}$, possibility of conductor's isolation combustion can be found using approximate formula (20) i.e. $Q_{1}(1)=0.99918 \cdot 10^{-6} \mathrm{In}$ our example $Q_{1}(1)$ value calculated by approximation formula coincides with $Q_{1}(1)$ value calculated by exact formula (6), i.e. $Q(1)=0.98245 \cdot 10^{-6}$.

\section{Conclusion}

Mathematical formulas $(6,12-14,17-20)$ that were synthesized in this article allow to assess apartment's fire safety during appearance of weakened sparkling disassembling dangerous contact joint. In addition, this methodology helped to reveal a fact, that usage of sparkprotecting devices with $\lambda_{2} \leq 0.21 /$ year and $\Theta_{2}=0.084$ 
year allows to guarantee a robust and good fire safety for power network.

This methodology has wide range of possible applications. It can be used for assessments of different spark protection systems during their development or installation. Moreover, technical maintenance of existing anti-fire systems made with the help of the proposed methodology can help to reveal more dangerous zones and prevent thousands of possible fires in living premises cause by sparkling in power networks.

The presented results were obtained as a part of scientific research according to the contract №11036GU/2016 "The development of power network condition controller with blackout prevention possibility" provided by Foundation for Assistance to Small Innovative Enterprises in Science and Technology (FASIE).

The presented results were obtained as a part of scientific researches according to the contract № 13.3746.2017.8.9 “The designing on the basis of systematic and logic probability evaluations of rational and economically proved structure of centralized, autonomous and combined power supply systems with high reliability and stability level with usage of alternative and renewable power sources for uninterrupted power supply of enterprises with continuous technological cycle".

\section{References}

1. S.V. Solyonyj O.P. Kovaliov, Scientifical papers of DonNTU, 10 (2011)

2. A.A. Vasin, et al. Shevchenko, FIPS 22 (2001)

3. P.A. Kuznetsov, A.V. Yudin, S.V. Solyonij, O.Ya. Solenaya, Herald of the Kharkiv National Technical Agricultural Univercity named after P. Vasilenko, 164 (2015)

4. S.V. Solyonyj, O.P. Kovaliov, G.V. Demchenko, M.O. Nagorniy, Unit for Power Network Protection (Ukrpatent, 2010)

5. B.N. Abramovich et al., Oil Industry, 6, 130-132 (2011)

6. GOST 12.1.004-91, Occupational safety standards system. Fire safety. General requirements, (Standards Publishing, 1992)

7. A. Dasgupta, P. Senasama, Design and Control of Matrix Converters (Springer, 2017)

8. H. Walter, B. Epple, Numerical Simulation of Power Plants and Firing Systems (Springer, 2017)

9. O.A. Shevchenko, Labor Protection, 20 (2003)

10. V. Khadkikar, M. Singh, A. Chandra, B. Singh, Joint International Conference on Power Electronics, Drives and Energy Systems (PEDES) 2010 Power India, 1-6 (2010)

11. S. Golestan, M. Monfared, and J. Guerrero, 4th Power Electronics, Drive Systems \& Technologies Conference (PEDSTC2013), 510-517 (2013)

12. Je.Ju. Brazilovich et al. Radio and Connection, 376 (1983)

13. A.P. Kovaliov, A.V. Shevchenko, I.V. Belousenko, Industrial Power Energy, 6 (1991)
14. Yu. Huang, P.M. Pardalos, Q.P. Zheng, Electrical Power Unit Commitment, (Springer, 2017)

15. B.N. Abramovich, Yu.A. Sychev, A.S. Mingazov, V.V. Polishuk, Oil Industry, 10, 126-127 (2013)

16. P.A. Kuznetsov, O.A. Stepanov, Aerospace MAI Journal, 4, 157-163 (2017)

17. F.Z. Peng, J.S Lai, IEEE Trans. on Inst. and Meast, 45 (1), 293-297 (1996)

18. B. Singh, K. Al-Haddad and A. Chandra, IEEE Trans. on Power Systems, 46 (5), 133-138 (1999)

19. A. Ferrero and G. Supert-Furga, IEEE Trans. Instrum. Meas., 40, 568-577 (1991)

20. B.N. Abramovich, Y.A. Sychev, International Journal of Applied Engineering Research, 11 (4), 2640-2645 (2016) 